\title{
Haemocompatibility of iron oxide nanoparticles synthesized for theranostic applications: a high-sensitivity microfluidic tool
}

\author{
Raquel O. Rodrigues • Manuel Bañobre-López • \\ Juan Gallo - Pedro B. Tavares • Adrián M. T. Silva • \\ Rui Lima $\mathbb{D} \cdot$ Helder T. Gomes
}

Received: 24 February 2016/ Accepted: 2 July 2016

(C) Springer Science+Business Media Dordrecht 2016

\begin{abstract}
The poor heating efficiency of the most reported magnetic nanoparticles (MNPs), allied to the lack of comprehensive biocompatibility and haemodynamic studies, hampers the spread of multifunctional nanoparticles as the next generation of therapeutic bio-agents in medicine. The present work reports the synthesis and characterization, with special focus on biological/toxicological compatibility, of superparamagnetic nanoparticles with diameter around $18 \mathrm{~nm}$, suitable for theranostic applications (i.e. simultaneous diagnosis and therapy of cancer). Envisioning more insights into the complex nanoparticle-red blood cells (RBCs) membrane interaction, the deformability of the human RBCs in contact with magnetic nanoparticles (MNPs) was assessed for the first time with a microfluidic extensional approach,
\end{abstract}

R. O. Rodrigues - H. T. Gomes

Laboratory of Separation and Reaction EngineeringLaboratory of Catalysis and Materials (LSRE-LCM), Polytechnic Institute of Bragança, Campus de Santa Apolónia, 5300-253 Bragança, Portugal

R. O. Rodrigues - A. M. T. Silva

Laboratory of Separation and Reaction EngineeringLaboratory of Catalysis and Materials (LSRE-LCM), Faculdade de Engenharia, Universidade do Porto, Rua Dr. Roberto Frias, 4200-465 Porto, Portugal

M. Bañobre-López · J. Gallo

Advanced (Magnetic) Theranostic Nanostructures Lab, INL-International Iberian Nanotechnology Laboratory, Av. Mestre José Veiga, 4715-330 Braga, Portugal and used as an indicator of haematological disorders in comparison with a conventional haematological test, i.e. the haemolysis analysis. Microfluidic results highlight the potential of this microfluidic tool over traditional haemolysis analysis, by detecting small increments in the rigidity of the blood cells, when traditional haemotoxicology analysis showed no significant alteration (haemolysis rates lower than $2 \%$ ). The detected rigidity has been predicted to be due to the wrapping of small MNPs by the bilayer membrane of the RBCs, which is directly related to MNPs size, shape and composition. The proposed microfluidic tool adds a new dimension into the field of nanomedicine, allowing to be applied as a highsensitivity technique capable of bringing a better

\section{P. B. Tavares}

CQVR-Centro de Química-Vila Real, Universidade de Trás-os-Montes e Alto Douro, 5000-801 Vila Real, Portugal

R. Lima $(\bowtie)$

Mechanical Engineering Department, MEtRiCS, University of Minho, Campus de Azurém, 4800-058 Guimarães, Portugal

e-mail: rl@dem.uminho.pt

\section{R. Lima}

CEFT-Centro de Estudos de Fenómenos de Transporte, Faculdade de Engenharia, Universidade do Porto, Rua Dr. Roberto Frias, 4200-465 Porto, Portugal 
understanding of the biological impact of nanoparticles developed for clinical applications.

Keywords Iron oxide nanoparticles . Haemocompatibility · RBCs deformation .

Microfluidic device · Magnetic hyperthermia · MRI · Superparamagnetism

\section{Introduction}

Iron oxide nanoparticles have attracted much attention in the last decades, especially for biomedical applications, due to their remarkable physicochemical properties acquired at the nanoscale. Superparamagnetism, high surface-to-volume ratio, high field irreversibility, high saturation magnetization, extra anisotropy contributions, biocompatibility and non-toxicity are some of these properties (Hervault and Thanh 2014; Rivas et al. 2012). Particularly, the possibility to be chemically functionalized with specific biocompatible targeting molecules provides them with the ability to selectively attach to cells or tissues. In addition, magnetic nanoparticles (MNPs) can be simultaneously used as contrast agents for magnetic resonance imaging (MRI) and for tumour therapy (i.e. magnetic hyperthermia and/or drug delivery), combining therapeutics and diagnosis, the so-called theranostics (Hervault and Thanh 2014). This combination is especially useful due to the enhanced permeability and retention effect (known as EPR effect), consisting of a selective accumulation of nanoparticles near the tumour tissues, normally surrounded by a high concentration of irregular and leaky blood vessels, and allowing the improvement of cancer diagnostic and specific treatments (Gallo et al. 2013).

Magnetic fluid hyperthermia (MFH) is considered a promising therapeutic technique for cancer treatment since it implements the remarkable nanoscale physicochemical properties of MNPs to generate heat under an alternating magnetic field. These nanoheaters can be designed to preserve healthy cells while destroying selectively tumoral cells, inherently more sensitive to mild temperature changes of $5-7^{\circ} \mathrm{C}$ above the body temperature (Jordan et al. 1999). Nevertheless, to get the desired therapeutic effect, MFH needs to fulfil several requirements that should be suitably balanced; MNPs should be designed small enough (i) to be able to cross the blood barrier and to penetrate into the tumour; (ii) to escape from the mononuclear phagocyte system (MPS) providing an enhanced blood circulation half-life; (iii) to have low sedimentation rates (i.e. high stability in suspension); and (iv) to show a good tissular diffusion (Mahmoudi et al. 2011; Rivas et al. 2012). Additionally, after the removal of the external magnetic field they should no longer show magnetization (negligible remanence and coercivity) to prevent their aggregation or the formation of clots in the blood circulation system (Bañobre-López et al. 2013; Obaidat et al. 2015). However, the reduction of the MNPs size is directly related to the reduction of the saturation magnetization $\left(M_{\mathrm{s}}\right)$ and, therefore, to the decrease of their heating efficiency (Bañobre-López et al. 2013; Deatsch and Evans 2014). In this context, a major concern in nanoparticle synthesis for biomedical applications is nanosize control and its manipulation changing synthetic parameters, such as $\mathrm{pH}$, temperature, ion species ratio and ionic strength, among others (Baumgartner et al. 2013; Hao et al. 2010). Despite the large number of reports on the development of MNPs for theranostic applications (Baumgartner et al. 2013; De Haas-Kock et al. 2009; Hayashi et al. 2013; Hocaoglu et al. 2015; Mahmoudi et al. 2011; Szekeres et al. 2015; Wang et al. 2015; Zavisova et al. 2015), just a few of them report biocompatibility studies of these MNPs (Grudzinski et al. 2013; Hocaoglu et al. 2015; Wang et al. 2015; Zavisova et al. 2015) and even less report their haemocompatibility (Hocaoglu et al. 2015; Wang et al. 2015). In fact, haemocompatibility determination is crucial to understand the real in vivo potential of any material envisioned for systemic administration, due to potential toxicological reactions (e.g. embolization, haemolysis and coagulation). In addition, haemocompatibility studies have been shown to be more sensitive than animal cell toxicity assays (Hocaoglu et al. 2015). Nevertheless, there is a lack of reports regarding the complex nanoparticle-RBCs membrane interaction, especially due to the small size of the nanoparticles, which makes very challenging to explore, even in vitro, the haemodynamic and the complex vascular environment (Tan et al. 2012). In this way, this paper presents for the first time a microfluidic tool based on a constriction channel capable of evaluating, in mimicked environments (close to in vivo microvessels), the biocompatibility and toxicity impact that biomedical-oriented MNPs 
present on human blood cells. Combining this microfluidic tool with a high-speed video microscopy system, multiple parameters (i.e. transit time, recovery time and cell deformability) that cannot be assessed via conventional approaches can be quantified with great detail. In the past, the first constriction channelbased microfluidic geometries were generally based on sudden and narrow channels, which were adopted to determine the rheological and hydrodynamic effect of these structures over the elasticity and deformation of the RBCs. Hence, it was observed that these constriction geometries had the ability to characterize the influence of possible diseases in the blood cells by determining differences in their natural deformations index (DI) (Abkarian et al. 2008). However, it was found that these close-fitting geometries in the form of long constrictions, which allow the uniaxial extensional motion and stretching of healthy blood cell along the flow direction, also promote the clogging of the channel when the blood cells increase their rigidity (Abkarian et al. 2008). To overcome this drawback, microfluidic devices with a hyperbolic channel based on extensional flow approach were adopted with great success by Lee et al. (2009), Yaginuma et al. (2013) and Faustino et al. (2014). In these pioneering works, the mechanical, rheological and haemodynamic environment created by the hyperbolic-shaped contractions were exhaustively tested, allowing to conclude that hyperbolic converging microchannel is a more accurate technique to determine the DIs of the flowing RBCs than shear flow-based methodologies. Moreover, it was demonstrated that the deformability of blood cells could be detailed and quantitatively described under a controlled homogeneous extensional flow, without the cell's tumbling and rotational motions usually observed in the shear flow-based methodologies (Lee et al. 2009; Oliveira et al. 2007; Yaginuma et al. 2013). Hence, this microfluidic methodology has proven to be the most adequate technique to gain insights into the DIs of RBCs as natural biomarkers to identify many pathologies (e.g. as malaria (Suwanarusk et al. 2004), diabetes (Sabo et al. 1993), coronary diseases (Yaylali et al. 2013), among others).

In line with aforementioned studies, the overriding aims of the present work are (1) the development of MNPs suitable for the diagnosis and treatment of cancer; (2) the determination of the haemocompatibility of the designed MNPs using a conventional analysis technique (i.e. haemolysis test); (3) the application of a microfluidic tool capable of bringing a new concept to assess with high accuracy the haemocompatibility and toxicity effect of MNPs developed for theranostic applications (i.e. hyperthermia, contrast enhancement agent for MRI, drug delivery systems, etc.). Moreover, and to best of our knowledge, this is the first time that an experimental microfluidic methodology is tested with the purpose of gaining new insights over the complex nanoparticleRBCs membrane interaction, mimicking micro-rheological conditions as those observed in in vivo microvascular environments.

\section{Experimental}

\section{Chemicals}

Ammonium hydroxide solution $\left(\mathrm{NH}_{4} \mathrm{OH}, 25\right.$ wt $\%$ in $\mathrm{H}_{2} \mathrm{O}$ ) was purchased from Merck (NJ, USA). Iron (II) chloride tetrahydrate $\left(\mathrm{FeCl}_{2} \cdot 4 \mathrm{H}_{2} \mathrm{O}, 99 \%\right)$, Dextran 40 $\left(\left(\mathrm{C}_{6} \mathrm{H}_{10} \mathrm{O}_{5}\right)_{\mathrm{n}}\right)$ and ethanol absolute $\left(\mathrm{C}_{2} \mathrm{H}_{6} \mathrm{O}, 99.8 \%\right)$ were purchased from Sigma-Aldrich (MO, USA). Iron (III) chloride hexahydrate $\left(\mathrm{FeCl}_{3} \cdot 6 \mathrm{H}_{2} \mathrm{O}, 97 \%\right)$ was supplied by Panreac (Barcelona, Spain). Alendronic acid and acetone were purchased from TCI Europe. Physiological salt solution (PSS) with $0.9 \% \mathrm{NaCl}$ was supplied by B. Braun Medical (Germany). All chemicals were of analytical grade and used as received without further purification. All aqueous solutions were prepared in deionized water.

Synthesis of magnetic nanoparticles (MNPs)

Briefly, $\quad \mathrm{FeCl}_{3} \cdot 6 \mathrm{H}_{2} \mathrm{O} \quad(134 \mathrm{mM}), \quad \mathrm{FeCl}_{2} \cdot 4 \mathrm{H}_{2} \mathrm{O}$ $(67 \mathrm{mM})$ and $\mathrm{NH}_{4} \mathrm{OH}(1 \mathrm{M})$ solutions were prepared in deionized water at room temperature. Two samples of magnetite were synthesized by alkaline co-precipitation, mixing in $100 \mathrm{~mL}$ of deionized water the iron precursors in the stoichiometric ratio 1:2 of iron (II) and iron (III) salts under vigorous magnetic stirring (C-Mag HS7, IKA) and heating the resultant mixture until the desired temperature $\left(30\right.$ and $\left.55^{\circ} \mathrm{C}\right)$. Then, the basic $\mathrm{NH}_{4} \mathrm{OH}(1 \mathrm{M})$ solution was added dropwise until $\mathrm{pH} \sim 9$, promoting the co-precipitation of the magnetite nanoparticles. The black solution formed in this process was maintained for $30 \mathrm{~min}$ at the same temperature and stirring conditions, ensuring the 
complete magnetite crystal formation. After that, the MNPs samples were subjected to repeated washing steps in deionized water to remove impurities; the last washing step was carried out with absolute ethanol and the final particles were dried at $60{ }^{\circ} \mathrm{C}$ overnight. The resulting materials were labelled as MAG30 and MAG55 depending on the used heating temperature (30 or $55^{\circ} \mathrm{C}$, respectively).

The colloidal stabilization of the ferrofluids is one of the most critical parameters to perform an accurate evaluation of the designed magnetic nanoparticles for theranostic applications (Ruiz et al. 2014). In general, the synthesized magnetic nanoparticles suffer from their strong high surface energy and hydrophobicity interactions, causing their agglomeration and fast sedimentation in the presence of an aqueous carrier fluid. In this sense, the functionalization of the developed magnetic nanoparticles with hydrophilic ligands represents a fundamental post-synthetic step for the further evaluation of the magnetic nanoparticles designed for theranostic applications. In this study, hydrophilic MNPs ferrofluids were obtained after addition of a solution of alendronic acid (Al, $50 \mathrm{mM}$, $\mathrm{pH}$ 10) into the colloidal suspensions of MAG30 or MAG55 $(1 \mathrm{mg} / \mathrm{mL})$, using a ratio $4: 1(\mathrm{v} / \mathrm{v})$. The mixtures were sonicated during $15 \mathrm{~min}$ and vigorously stirred for $48 \mathrm{~h}$ at room temperature. These resulting suspensions were centrifuged at $6000 \mathrm{rpm}$ for $5 \mathrm{~min}$ (Allegra 64R, Beckman Coulter) and washed with acetone to remove free alendronate from the coated MNPs. The purification procedure was repeated twice. The resulting MNPs@Al (MAG30@Al and MAG55@Al)were dispersed in water, sonicated for $30 \mathrm{~s}$ and stored at room temperature.

\section{Characterization of magnetic nanoparticles}

\section{Size, crystal structure and chemical composition}

Transmission electron microscopy (TEM, LEO/ZEISS 906E) was used to analyse individually the morphology and particle size distribution, MAG30 and MAG55, employing an ImageJ software (1.46r, NIH). For each sample, 120 individual MNPs were randomly measured and the results shown as mean. The crystalline phases of these materials were analysed by X-ray diffraction (XRD) in a PAN'alytical X'Pert PRO equipment $(\mathrm{CuK} \alpha$ radiation) equipped with $\mathrm{X}^{\prime}$ Celerator detector and secondary monochromator, in $\theta / 2 \theta$ geometry. The diffractograms were analysed by Rietveld refinement using PowderCell software. Crystallite sizes were calculated applying Williamson-Hall model to refinement results. An inductively coupled plasma-optical emission spectrometer (ICP-OES, ICPE-9000, Shimadzu) was employed to determine the iron content on the MNPs@Al hydrophilic samples.

\section{Magnetic and hyperthermia properties}

The magnetic properties of the powder samples, MAG30 and MAG55, were investigated with a superconducting quantum interference device (SQUIDVSM) magnetometer from Quantum Design. Hysteresis curves were recorded for magnetic fields between $-20 \mathrm{kOe}$ and $+20 \mathrm{kOe}$ at room temperature $(300 \mathrm{~K})$. The temperature-dependent magnetization was recorded under a magnetic field of $50 \mathrm{Oe}$ in the temperature range between 2 and $300 \mathrm{~K}$, in both zerofield-cooled (ZFC) and field-cooled (FC) samples. The MNPs@Al heating efficiencies were evaluated in a hyperthermia equipment (DM 100 system, nB nanoscale Biomagnetics) using $1 \mathrm{~mL}$ of MNPs@ $\mathrm{Al}$ water

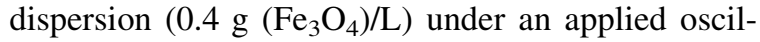
lating magnetic field of $15.95 \mathrm{kA} / \mathrm{m}$ at a resonant frequency of $688 \mathrm{kHz}$. The temperature increase was measured with an optical fibre and recorded as a function of the time for $10 \mathrm{~min}$.

\section{Magnetic resonance relaxivity studies}

The MRI contrast enhancement efficiency of MNPs@Al was studied by the determination of the $T_{1}$ (longitudinal) and $T_{2}$ (transversal) relaxation times with a fixed field relaxometer (Minispec NMR spectrometer mq60, Bruker Instruments) at a magnetic field strength of $1.41 \mathrm{~T}$. To this purpose, three different $\mathrm{Fe}$ concentrated solutions were prepared of each sample(MAG30@Al and MAG55@Al), with Fe concentrations of $0.00,0.04$, and $0.08 \mathrm{mM}$. The $r_{1}$ and $r_{2}$ relaxivities were determined from the slopes of a linear fitting of $1 / T_{1}$ or $1 / T_{2}$ versus the Fe content of the samples.

\section{Haemolysis analysis}

Haemolysis induced by nanoparticles treatment was assessed photometrically by a UV-Vis spectrophotometer (T70 spectrometer, PG Instruments Ltd.). 
Fig. 1 Microfluidic device fabricated in PDMS with a hyperbolic-shaped contraction to assess the haemocompatibility of the RBCs in contact with MNPs

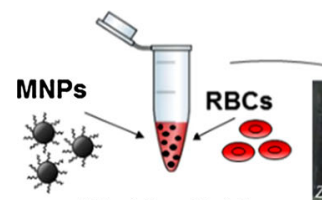

Working fluid
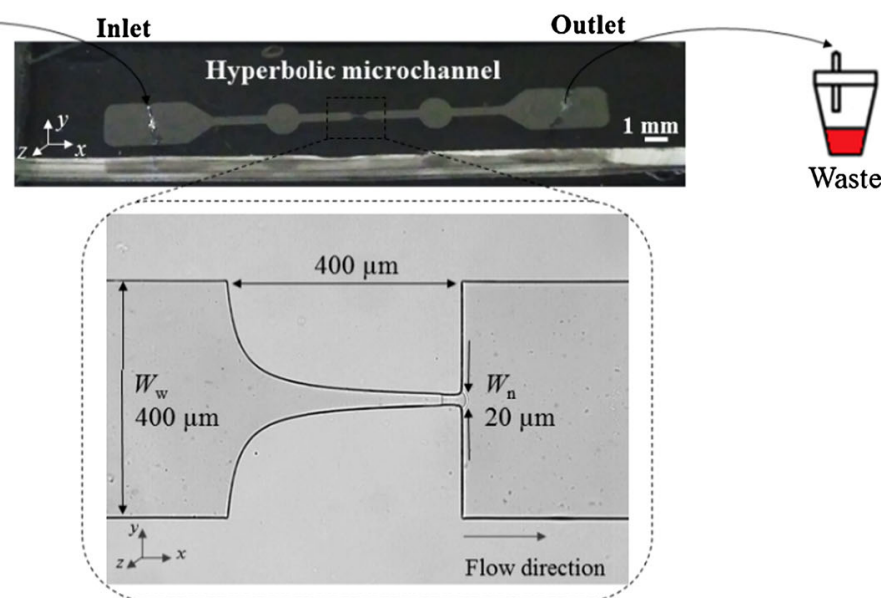

Lima et al. (2008). Figure 1 shows the main channel of the microfluidic device that was designed to have the following dimensions: $20 \times 400 \times 26,389 \mu \mathrm{m}$ (height $\times$ width $\times$ length).

Furthermore, in the centre of this main channel, a hyperbolic-shaped contraction with a Hencky strain of $\sim 3$ (also called as logarithmic strain, $\varepsilon_{\mathrm{H}}=\ln \left(W_{\mathrm{w}} / W_{\mathrm{n}}\right)$ (Oliveira et al. 2007)), was designed to have the following dimensions: $400 \mu \mathrm{m}$ of length and widths of 400 and $20 \mu \mathrm{m}$, respectively, at the wide $\left(W_{\mathrm{w}}\right)$ and narrow sizes $\left(W_{\mathrm{n}}\right)$. As a consequence, the high Hencky strain imposed on this geometry allows an almost linear velocity increase with the axial position, $x$, without suffering entrance or exit effects (Oliveira et al. 2007).

The microscopy system used in this work consisted on an inverted microscope (IX71, Olympus) combined with a high-speed camera (Fastcam SA3, Photron, USA). In the inverted microscope, the PDMS microchannel was placed and fixed, and the flow rate of the working fluids kept constant at $5 \mu \mathrm{L} / \mathrm{min}$ by means of a syringe pump (PHD Ultra, Harvard Apparatus, USA) with a $2 \mathrm{~mL}$ syringe (Terumo, Japan). At the same time, the images of the flowing blood cells, at the established flow rate, were captured by the high-speed camera with a frame rate of 2000 frames/s and a shutter speed ratio of $1 / 75,000$. These capture parameters allowed the minimization of cells dragging caused by the high flow rate.

Table 1 shows the experimental parameters used to perform the haemodynamic study of RBCs as natural biophysical markers to evaluate the MNPs biocompatibility, by assessing their DIs in the described hyperbolic channel.
The polydimethylsiloxane (PDMS) microchannels
evaluated in this work were fabricated using a softlithographic technique, as previously reported by 
Table 1 Experimental parameters used to perform the haemodynamic study of RBCs, as natural biophysical markers to evaluate the MNPs biocompatibility by assessing their DIs

\begin{tabular}{|c|c|}
\hline \multicolumn{2}{|l|}{ Experimental parameters } \\
\hline Maximum width of the microchannel & $400 \mu \mathrm{m}$ \\
\hline Minimum width of the microchannel & $20 \mu \mathrm{m}$ \\
\hline Total length of the contraction region & $400 \mu \mathrm{m}$ \\
\hline Depth of the microchannel & $20 \mu \mathrm{m}$ \\
\hline Inlet flow rate & $5 \mu \mathrm{L} \min ^{-1}$ \\
\hline Mean velocity of the fluid & $5.21 \mathrm{~mm} \mathrm{~s}^{-1}$ \\
\hline Mean diameter of the human $\mathrm{RBC}$ at rest & $8 \mu \mathrm{m}$ \\
\hline Shear viscosity of the Dextran 40 & $4.5 \times 10^{-3} \mathrm{~Pa} \mathrm{~s}^{-1}$ \\
\hline Density of the Dextran 40 & $1046 \mathrm{~kg} \mathrm{~m}^{-3}$ \\
\hline Haematocrit of the working fluid & $2 \%$ \\
\hline MNPs concentration & 17.4 and $34.8 \mu \mathrm{g}$ Magnetite $/ \mathrm{mL}$ \\
\hline Temperature of the working fluid & $37^{\circ} \mathrm{C}$ \\
\hline Magnification (M) & $20 \times($ optical zoom $1.6 \times)$ \\
\hline Frame rate & 2000 frames s $^{-1}$ \\
\hline Exposure time & $500 \mu \mathrm{s}$ \\
\hline
\end{tabular}

\section{Working fluid protocol}

A Dextran 40 solution (10\%,w/v) containing $2 \%$ of haematocrit (RBCs, v/v) was used as the working fluid to perform the deformability tests in the microfluidic devices. The amount of MNPs@ Al was studied at 0.0, 17.4 and $34.8 \mu \mathrm{g}\left(\mathrm{Fe}_{3} \mathrm{O}_{4}\right) / \mathrm{mL}$. The working fluid prepared without MNPs@Al $\left(0.0 \mu \mathrm{g}\left(\mathrm{Fe}_{3} \mathrm{O}_{4}\right) / \mathrm{mL}\right)$ was used as positive control.

Briefly, venous human blood samples from healthy donors were collected into $2.7 \mathrm{~mL}$ tubes (SMonovette $^{\circledR}$, Sarstedt) containing EDTA to avoid coagulation, and centrifuged at $2500 \mathrm{rpm}$ for $10 \mathrm{~min}$ at $4{ }^{\circ} \mathrm{C}$. The buffy coat and plasma were removed and the packed RBCs re-suspended and washed twice in PSS with $0.9 \% \mathrm{NaCl}$, in a ratio $1: 1(\mathrm{v} / \mathrm{v})$. The final working fluid was prepared immediately before the experiments by mixing together the collected RBCs with the desired amount of MNPs@Al in the Dextran 40 solution (10\%,w/v). Dextran 40 solution was used as carrier fluid instead of the blood plasma to avoid the clotting and jamming of the blood cells in the in vitro microfluidic studies, which are generally promoted by some components, i.e. immunoglobulins, clotting factor, albumin and fibrinogen proteins. The working fluid samples were incubated at $37{ }^{\circ} \mathrm{C}$ during 15,30 , and $60 \mathrm{~min}$ in a shaking water bath, allowing the evaluation of the time-contact effect between the MNPs and the deformability of RBCs.

\section{Image analysis}

The experimental images recorded in each test were transferred to a computer, processed and analysed by an image handling software, ImageJ (1.46r, NIH, USA). Using this software, DI of the blood cells was calculated as previously reported by Pinho et al. (2013), using Eq. (2) (Yaginuma et al. 2013):

$\mathrm{DI}=L_{\text {major }}-L_{\text {minor }} / L_{\text {major }}+L_{\text {minor }}$

For each deformability test performed with three amounts of MNP@ $\mathrm{Al}\left(0.0,17.4\right.$ and $34.8 \mu \mathrm{g}\left(\mathrm{Fe}_{3} \mathrm{O}_{4}\right)$ / $\mathrm{mL}$ ), the DI of 112 red blood cells in two independent experiments was assessed along the microfluidic channel with a hyperbolic-shaped contraction followed by a sudden expansion.

\section{Statistical analysis}

The microfluidic results were displayed as mean $\pm \mathrm{s}-$ tandard deviation using two independent experiments ( $n=112$ red blood cells). The statistical analysis was performed with the Student's $t$ test for one-tailed distribution with a significance level of $p<0.05$, represented as asterisks $(*)$ in comparison with the control test. All statistical analyses were performed using Microsoft Office Excel (version Professional Plus 2013). 
Ethical approval

Ethical approval was given by the medical ethics committee of the Unidade Local de Saúde do Nordeste, E.P.E. (Bragança, Portugal).

\section{Results and discussion}

Characterization of the developed MNPs

\section{Size, crystal structure and chemical composition}

The results obtained by TEM analysis of MAG30 and MAG55 are shown in Fig. 2, revealing that the magnetic core of the synthesized nanoparticles is nearly spherical for both samples. Nevertheless, the increase of the synthesis temperature leads to a growth in the average diameter of the magnetic core, from 11.1 (MAG30) to $17.7 \mathrm{~nm}$ (MAG55), which is in good agreement with the crystallite sizes obtained by the XRD analysis (Table 2), as expected for particle sizes within the single-domain superparamagnetic region.
Additionally, the determination of the nanoparticle size distribution reveals that not only the average nanoparticle diameter grows as synthesis temperature increases, but also the nanoparticle size polydispersion. Nevertheless, for the higher synthesis temperature used in this study $\left(55^{\circ} \mathrm{C}\right)$, none of the measured MNPs was found to be larger than $50 \mathrm{~nm}$.

All the reflection peaks of the XRD patterns of MAG30 and MAG55 were indexed by considering a magnetite phase. No reflections peaks were observed as impurities or secondary phases. The cubic cell lattice parameter resulted to be $a \sim 8.37 \AA$, in good agreement with the values reported for nanosized magnetite (Aphesteguy et al. 2015). Also, Table 2 shows the crystallite size, which was calculated from the 5 most intense peaks of the XRD patterns through the Williamson-Hall formula (Khorsand et al. 2011). For comparison reasons, the particle diameter observed by TEM is also shown that resulted to be very similar to that obtained from the XRD data, as expected for particle sizes within the single-domain superparamagnetic region. Thus, these results enable the co-precipitation method under mild experimental conditions, as a suitable synthesis method to ensure the
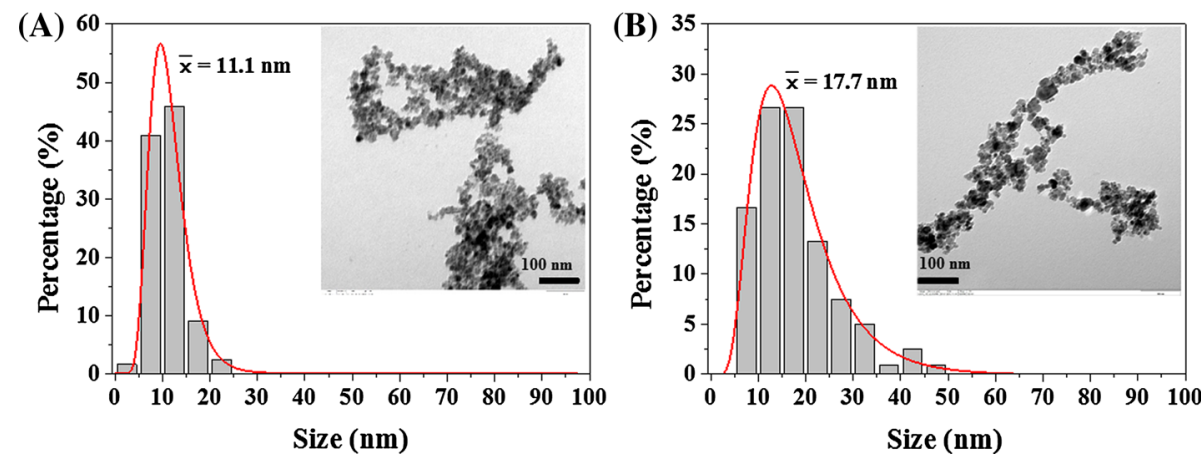

Fig. 2 Histogram and mean diameter $(\bar{X})$ of the nanoparticles following the Log-normal distribution of a MAG30 and b MAG55. Inset shows the corresponding TEM images

Table 2 Physico-chemical properties of the synthesized nanoparticles: diameter determined by TEM $\left(d_{\text {TEM }}\right)$, crystallite size calculated from XRD $\left(d_{\mathrm{hkl}}\right)$, lattice constant and crystalline core phase obtained by XRD analysis

\begin{tabular}{|c|c|c|c|c|c|}
\hline Sample & Synthesis method & $d_{\text {TEM }}(\mathrm{nm})^{\mathrm{a}}$ & $d_{\mathrm{hkl}}(\mathrm{nm})^{\mathrm{b}}$ & Lattice constant, a $(\AA)$ & Crystalline core phases, $\%(\mathrm{v} / \mathrm{v})$ \\
\hline MAG30 & Co-precipitation, $30^{\circ} \mathrm{C}$ & 11.1 & 12.8 & 8.3652 & $100 \%$ Magnetite $\left(\mathrm{Fe}_{3} \mathrm{O}_{4}\right)$ \\
\hline MAG55 & Co-precipitation, $55^{\circ} \mathrm{C}$ & 17.7 & 18.5 & 8.3653 & $100 \%$ Magnetite $\left(\mathrm{Fe}_{3} \mathrm{O}_{4}\right)$ \\
\hline
\end{tabular}

${ }^{a}$ Estimated using ImageJ software as mean $(n=120)$

b Calculated using the Williamson-Hall equation (Khorsand et al. 2011) 
Table 3 Magnetic properties of the $\mathrm{Fe}_{3} \mathrm{O}_{4}$ nanoparticles synthesized by co-precipitation at different synthesis temperatures (30 and $\left.55^{\circ} \mathrm{C}\right)$ : saturation magnetization $\left(M_{\mathrm{s}}\right)$, coercivity $\left(H_{\mathrm{c}}\right)$, saturation remanence $\left(M_{\mathrm{r}}\right)$, SAR and ILP

\begin{tabular}{llllll}
\hline Sample & $M_{\mathrm{s}}\left(\mathrm{emu} / \mathrm{g} \mathrm{Fe}_{3} \mathrm{O}_{4}\right)$ & $H_{\mathrm{c}}(\mathrm{Oe})$ & $M_{\mathrm{r}}\left(\mathrm{emu} / \mathrm{g} \mathrm{Fe}_{3} \mathrm{O}_{4}\right)$ & $\mathrm{SAR}^{\mathrm{a}}\left(\mathrm{W} / \mathrm{g} \mathrm{Fe}_{3} \mathrm{O}_{4}\right)$ & $\mathrm{ILP}^{\mathrm{b}}\left(\mathrm{nHm}^{2} / \mathrm{kg}\right)$ \\
\hline MAG30 & $56.19 \pm 0.02$ & 33.65 & 3.77 & 100.18 & 0.57 \\
MAG55 & $77.68 \pm 0.03$ & 18.33 & 1.94 & 565.68 & 3.23
\end{tabular}

${ }^{a}$ Specific absorption rate (SAR) calculated for $H=15.95 \mathrm{kA} / \mathrm{m}, f=688 \mathrm{kHz}, C=0.40 \mathrm{~g}\left(\mathrm{Fe}_{3} \mathrm{O}_{4}\right) / \mathrm{L}$

b Intrinsic loss power (ILP) independent of the magnetic field parameters, allowing direct comparison between experiments under different experimental conditions

Fig. 3 Magnetic characterization of MAG30 and MAG55. a Hysteresis loops at room temperature $(300 \mathrm{~K})$ up to $\pm 20 \mathrm{kOe}$; the inset is a zoom in the lowfield region; b ZFC-FC magnetization curves measured at 50 Oe
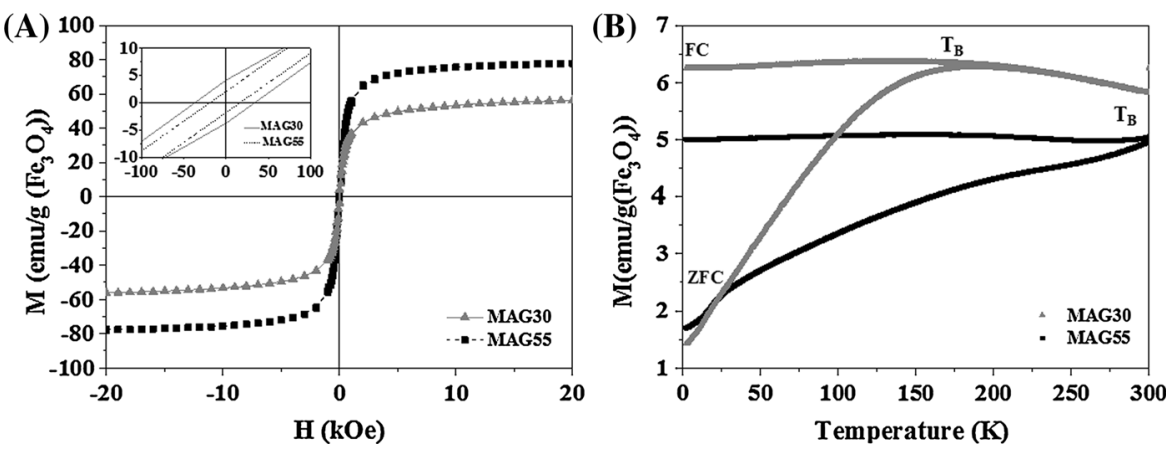

presence of a single-phase (magnetite), which is ideal for biomedical applications.

\section{Magnetic and hyperthermia properties}

The main magnetic parameters of MAG30 and MAG55, namely saturation magnetization, coercivity and remanence, were calculated from the analysis of the magnetization curves as a function of the applied magnetic field obtained for powdered samples (see Table 3; Fig. 3). The specific absorption rate (SAR, W/g $\left.\left(\mathrm{Fe}_{3} \mathrm{O}_{4}\right)\right)$ values were calculated according to Eq. (3) (Szekeres et al. 2015):

$\mathrm{SAR}=\left(C m_{\text {water }} / m_{\mathrm{Fe} 3 \mathrm{O} 4}\right)(\Delta T / \Delta t)$

where $C$ is the specific heat capacity of the medium $\left(C_{\text {water }}=4.186 \mathrm{~J} \mathrm{~g}^{-1}{ }^{\circ} \mathrm{C}^{-1}\right), m_{\text {water }}$ and $m_{\mathrm{Fe} 3 \mathrm{O} 4}$ are the masses of the medium and magnetite, respectively, and $\Delta T / \Delta t$ is the initial temperature rate. In addition, the intrinsic loss power (ILP), independent of the magnetic field amplitude and frequency used in the experiment and that allows for a direct comparison between different reported values, was calculated using Eq. (4) (Kolen'ko et al. 2014):

$\mathrm{ILP}=\mathrm{SAR} / H^{2} f$ where SAR is the specific absorption rate given by Eq. (3), $H$ is the field amplitude and $f$ is the frequency used in the hyperthermia experimental setup.

Both MAG30 and MAG55 hysteresis loops show a superparamagnetic-like behaviour. Whereas MAG30 $(11.1 \mathrm{~nm}$ of particle size) showed a saturation magnetization, $M_{\mathrm{s}}$, of $56 \mathrm{emu} / \mathrm{g}$, the increase of the synthesis temperature in MAG55 led to an increase of the particle size $(17.7 \mathrm{~nm})$ that subsequently resulted in an increase of $M_{\mathrm{s}}$, which is near to the bulk value of magnetite, i.e. $90 \mathrm{emu} / \mathrm{g}$ (Lu et al. 2007). Nevertheless, MAG55 exhibits lower remanence $\left(M_{\mathrm{r}}\right)$ and coercive forces $\left(H_{\mathrm{c}}\right)$ at room temperature compared to MAG30, despite the higher particle size of the individual particles (see Table 2). This unlike result has been previously reported (Kolen'ko et al. 2014), and explained to be caused by the effect of the dipolar magnetic interactions caused by the presence of clustering in small nanoparticles.

On the other hand, the zero-field-cooling-fieldcooling (ZFC-FC) magnetization curves shown in Fig. 3 indicate a shift of the blocking temperature $\left(T_{\mathrm{B}}\right)$, defined as the temperature above which the MNPs show superparamagnetic behaviour (Obaidat et al. 2015), from 175 to $300 \mathrm{~K}$ (or higher) for MAG30 
and MAG55, respectively. In both cases, the flatness of the FC curve below $T_{\mathrm{B}}$ is indicative of significantly strong dipolar magnetic particle interactions. Hence, the confirmation of a superparamagnetic state for both MAG30 and MAG55 at room temperature makes these samples suitable for MRI and hyperthermia applications.

The hyperthermia performance of MAG30@Al and MAG55@Al nanoparticles was determined in water solution at a concentration of $0.40 \mathrm{~g}\left(\mathrm{Fe}_{3} \mathrm{O}_{4}\right) / \mathrm{L}$. Both nanoparticle dispersions were evaluated in terms of specific absorption rate (SAR) and independent loss power (ILP), which are shown in Table 3. Figure 4 shows the temperature profiles as a function of time for both samples under the same hyperthermia conditions $(H=15.95 \mathrm{kA} / \mathrm{m} ; f=688 \mathrm{kHz})$.

As expected, SAR increases with increasing size of the magnetic core of the iron oxide nanoparticles. Indeed, many works compiled by Deatsch and coworkers in 2014, pointed to the optimal diameter for magnetite nanoparticles be applied in magnetic hyperthermia, fall somewhere between 12 and $20 \mathrm{~nm}$. However, the comparison between different studies is very difficult since SAR results strongly depend on the magnitude and frequency of the applied magnetic field. Thus, to overcome this issue, ILP was proposed as a measure of heating efficiency that normalizes SAR with respect to field strength, $H$, and frequency, $f$. ILP results revealed a remarkable heating efficiency for the MAG55 nanoparticles $\left(3.23 \mathrm{nHm}^{2} / \mathrm{kg}\right)$ when compared with ILP values found for coated magnetite (e.g. mPEGs, Chitosan, 2,3-Dimercaptosuccinic acid,

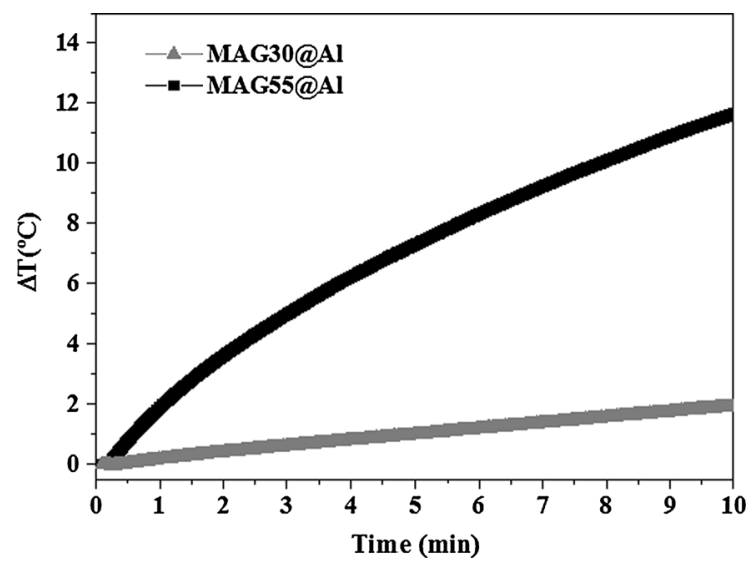

Fig. 4 Temperature versus time curves for MAG30@ $\mathrm{Al}$ and MAG55@Al water colloidal dispersions at $0.40 \mathrm{~g}\left(\mathrm{Fe}_{3} \mathrm{O}_{4}\right) / \mathrm{L}$, under an oscillating magnetic field of $15.95 \mathrm{kA} / \mathrm{m}$ and $688 \mathrm{kHz}$
(3-Amino-propyl)triethoxysilane, Dextran, among others) (Hervault and Thanh 2014; Sharifi et al. 2012), or even reported commercial ferrofluids, which are in the range $0.20-3.10 \mathrm{nHm}^{2} / \mathrm{kg}$ (Presa et al. 2012). In fact, it should be noted that the hyperthermia tests performed in this work were made with an extremely low concentration of colloidal suspension $(0.40 \mathrm{~g}$ $\left.\left(\mathrm{Fe}_{3} \mathrm{O}_{4}\right) / \mathrm{L}\right)$, which is at least 10 times lower than the typical values used in other published magnetic hyperthermia tests (Presa et al. 2012; Szekeres et al. 2015). Nevertheless, the sample MAG55@Al showed a temperature increment of $12{ }^{\circ} \mathrm{C}$, after $10 \mathrm{~min}$ of exposure to the applied magnetic field. Thus, the derived good ILP result obtained for MAG55@Al is likely due to the combination of narrow size distribution of the synthesized MNPs, good colloidal stability and strong magnetic response caused by the Néel and Brownian power loss mechanisms, dominant and comparable in superparamagnetic nanoparticles.

\section{Magnetic resonance relaxivity studies}

The contrast enhancement efficiency of MNPs@Al was evaluated in water through relaxivity measurements by plotting the relaxation rates $\left(R_{1}=1 / T_{1}\right.$ and $R_{2}=1 / T_{2}$ ) of water protons in the presence of the nanoparticles, against the $\mathrm{Fe}$ content. Upon linear fitting, the relaxivity values, $r_{2}$ and $r_{1}$, were obtained from the respective slope and are listed in Table 4, as well as the corresponding $R^{2}$ of the linear fit, which showed good fitting results.

Both MAG30@Al and MAG55@Al ferrofluidic samples showed a similar $r_{1}$ of $\sim 4 \mathrm{mM}^{-1} \mathrm{~s}^{-1}$, which is higher than that of reported superparamagnetic iron oxide nanoparticles (Szekeres et al. 2015). More importantly, enhanced $r_{2}$ values of $248.17 \mathrm{mM}^{-1} \mathrm{~s}^{-1}$ for MAG55@Al and $226.08 \mathrm{mM}^{-1} \mathrm{~s}^{-1}$ for sample MAG30@Al were achieved, which are in good agreement with other results with surface modified $\mathrm{Fe}_{3} \mathrm{O}_{4}$ nanoparticles (Barick et al. 2014), and far superior than currently available intravenous iron oxide nanoparticle contrast agents (Weinstein et al. 2010). Moreover, and as expected, these results indicate that the relaxivity values $r_{1}$ and $r_{2}$ increase with the MNPs growth, very likely due to the increase in saturation magnetization.

In MRI, two main families of contrast agents can be used, $T_{1}$ or agents that affect mainly the longitudinal relaxation (positive contrast, brightening of the 
Table 4 Longitudinal $\left(r_{1}\right)$ and transverse $\left(r_{2}\right)$ relaxivity of MAG30@Al and MAG50@Al. The corresponding coefficient of the linear fit $\left(R^{2}\right)$ and relaxivity ratio $\left(r_{2} / r_{1}\right)$ is also shown

\begin{tabular}{llllll}
\hline Sample & $r_{2}^{\mathrm{a}}\left(\mathrm{mM}^{-1} \mathrm{~s}^{-1}\right)$ & $R^{2}\left(\mathrm{r}_{2}\right)$ & $r_{1}^{\mathrm{a}}\left(\mathrm{mM}^{-1} \mathrm{~s}^{-1}\right)$ & $R^{2}\left(\mathrm{r}_{1}\right)$ & $r_{2} / r_{1}$ \\
\hline MAG30@Al & 226.08 & 0.9986 & 4.02 & 0.9990 & 56.24 \\
MAG55@Al & 248.17 & 0.9992 & 4.42 & 0.9999 & 56.15 \\
\hline
\end{tabular}

${ }^{a}$ Relaxivity values measured at $B=1.41 T$ and $37^{\circ} \mathrm{C}$

image) and $T_{2}$ or agents that have an effect predominantly on the transverse relaxation (negative contrast, darkening of the image) (Gallo et al. 2014). The $r_{2} / r_{1}$ ratio is considered a suitable indicator to assess the efficiency of MNPs as a $T_{1}$ or a $T_{2}$ contrast agent. The $r_{2} / r_{1}$ ratio of MAG30 and MAG55 resulted to be higher than that of some commercial iron oxide nanoparticles contrast agents (Saraswathy et al. 2014; Weinstein et al. 2010), indicating that both samples are promising candidates for high-efficiency $T_{2}$-weighted MR imaging.

\section{Haemolysis analysis}

Haemolysis analysis is one of the most common studies performed to evaluate the haemocompatibility of nanoparticles for biomedical applications (Lin et al. 2012; Wang et al. 2015), especially when they are intended to be applied directly into the blood stream. In fact, previous in vitro studies demonstrated that several nanoparticles induced haemolysis, the extent of the effect being directly related to particle size, surface properties and experimental conditions (Lin et al. 2012).

In the present study, two different contents of MAG55@Al (the sample that shows better performance in both MRI and MFH) were suspended in diluted whole blood and tested, 17.4 and $34.8 \mu \mathrm{g}$ $\left(\mathrm{Fe}_{3} \mathrm{O}_{4}\right) / \mathrm{mL}$. The selection of these concentrations was based on the recommended dosage $(15 \mu \mathrm{mol}(\mathrm{Fe}) / \mathrm{kg}$ of individual) for the commercial superparamagnetic iron oxide-based MRI enhancer (ENDOREM ${ }^{\circledR}$, Guerbet S.A.) in individuals with a weight of 75 and $150 \mathrm{~kg}$, respectively. This choice was made since there are no guidelines available concerning the optimal concentration of iron oxide nanoparticles to be applied systemically for magnetic hyperthermia treatment. Thus, the absorbance results of the supernatant fluids, treated with different concentrations, were compared and normalized with the result from lysed RBCs in the presence of deionized water (taken as $100 \%$ lysed, negative control) and the blood in PSS buffer without MNPs (taken as $0 \%$ lysed, positive control), using for this purpose Eq. (1).

According to the standard ISO 10993-4 for the evaluation of biological medical devices suitable for blood contact, the material should have haemolysis rates lower than $5 \%$ (Wang et al. 2015). In this work, the haemolysis rates were found to be lower than $2 \%$ for all tested concentrations. More precisely, it was found to be $1.08 \pm 0.73 \%$, for a low content of nanoparticles $\left(17.4 \mu \mathrm{g}\left(\mathrm{Fe}_{3} \mathrm{O}_{4}\right) / \mathrm{mL}\right)$ and $1.63 \pm 0.28 \%$ for the highest $\left(34.8 \mu \mathrm{g}\left(\mathrm{Fe}_{3} \mathrm{O}_{4}\right) / \mathrm{mL}\right)$. Therefore, and according to the guide for the evaluation of biological materials, the synthesized MNPs developed in this work proved to fulfil the requirements of the haemolysis test to be applied as medical materials for blood contact, even at the highest concentration tested.

\section{Microfluidic studies}

In the literature, only a few studies can be found reporting the influence of nanoparticles into the microrheological properties of the human RBCs, or vice versa, for biomedical application purposes (Mayer et al. 2009; Müller et al. 2014; Thomas et al. 2014). From those, some of them only report numerical simulation results without comparison with experimental tests (Müller et al. 2014; Thomas et al. 2014). The main reason for this lack of in vitro studies may be the mandatory requirement of multidisciplinary research teams and high-tech laboratory facilities. Nevertheless, to promote the application of multifunctional nanomedicine based on nanoparticles, many microfluidic, mechanistic and toxicological milestones have to be accomplished in the near future. Based on the expectation to provide mechanistic insights on nanomedicines designed for theranostic applications, a microfluidic tool was applied for the first time to determine the impact of MNPs on the 

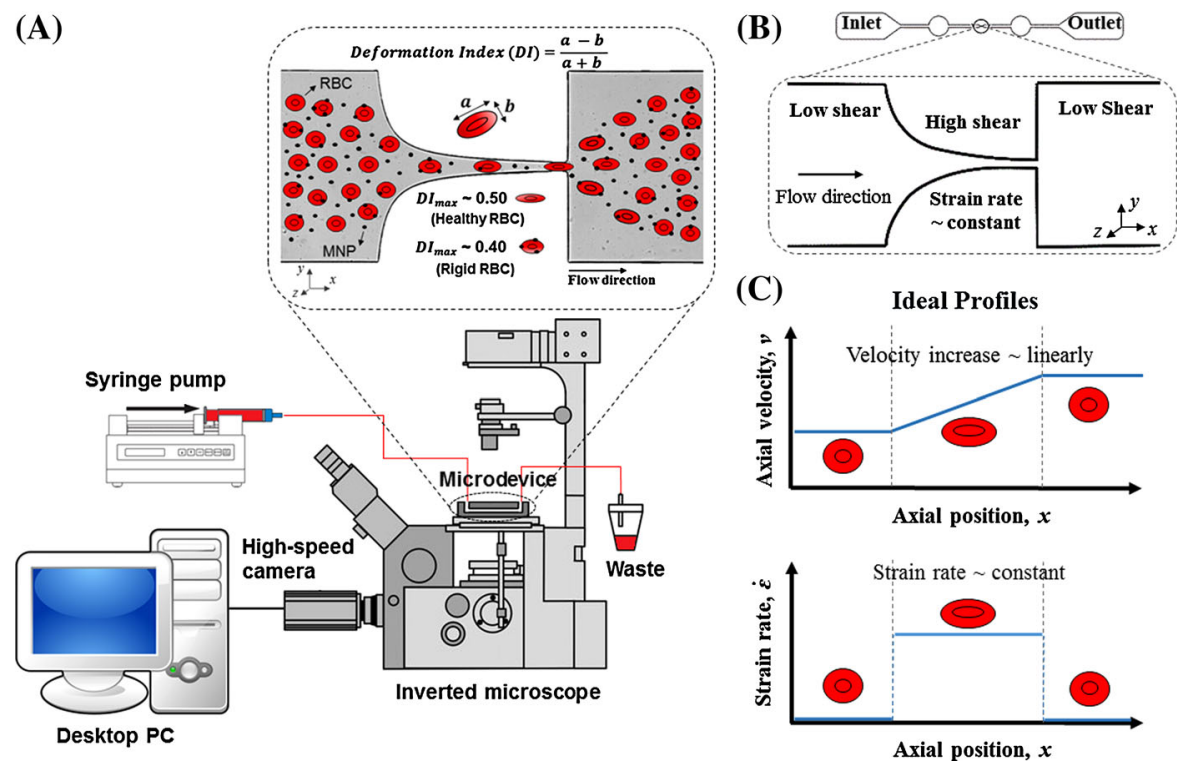

Fig. 5 Microfluidic studies for the haemocompatibility of the RBCs in contact with MNPs. a Schematic view of the experimental setup; b Microchannel device geometry with the zoom of the hyperbolic channel; c Graphical representation of

RBCs membranes (the major component of the blood and the principal actor in micro-rheology).

The abnormal RBCs rigidity has been already related to several diseases, pointing to the modification of the membrane structural components (e.g. skeletal filaments, transmembrane proteins and fatty acids) (Kang et al. 2016; Zheng et al. 2012) and cytoplasmatic content as the main reasons for this abnormal rigidity change (Rodrigues et al. 2015). As a result, rigid RBCs can cause phenomena of clotting in the micro-circulatory vessels (namely, capillaries, arterioles and venules), leading to the blocking of the normal blood flow in microcirculation (Kang et al. 2016), which in the worst scenarios can cause ischaemia in the tissues and/or lead to episodes of cerebrovascular accidents (Yaginuma et al. 2013). Hence, these blood cells have the potential to be used as natural biophysical markers (Kang et al. 2016; Zheng et al. 2012) without the costly labelling and sample handling that is common for most biochemical markers (Gossett et al. 2012). In this study, and for the first time, a high-sensitivity microfluidic tool capable to assess the deformability of blood cells was used to evaluate the impact of nanoparticles on the human RBCs. This methodology comprises a microfluidic device having a hyperbolic-shaped contraction the fluid-induced condition profiles that occur in the hyperbolic channel, which has proven to be a more efficient way for the determination of the DI of the RBCs

microchannel and a high-speed video microscopy system. This combined system enables the recording, in real time, of the biomechanical microfluidic phenomena, which can be then further analysed to obtain valuable toxicological data.

Figure 5 shows a schematic view of the experimental setup, the microfluidic device with the hyperbolic channel and the fluid-induced deformation profiles occurring at the hyperbolic channel, making this technique ideal for DI assessment.

Figure 5a represents the high-speed video microscopy system used to perform the microfluidic studies. Moreover, Fig. 5b, c shows the fluid-induced deformation profiles that attest that the hyperbolic channel approach is ideal for the assessment of the RBC's DI. As previously referred, this extensional flow technique has already been shown to be more efficient in deforming RBCs and assess their DI than other techniques based on the shear flow (Lee et al. 2009; Oliveira et al. 2007; Yaginuma et al. 2013). In this sense, this extensional flow technique was used for the first time as a microfluidic concept capable of gaining new insights over the complex nanoparticle-RBCs interaction designed for theranostic applications. The main reason for this ability is the promotion of the homogeneous extensional flow along the centreline of 
the hyperbolic channel with a high Hencky strain. In this type of microfluidic devices, the velocity increases almost linearly and the strain rate is maintained approximately constant, even with the increment of the shear rate (Oliveira et al. 2007). As a result, this reported technique allows the deformation of the blood cells along the microchannel, causing the RBC stretching by the larger fluid shear stress, without tumbling and rotational motions of the cells, providing the most accurate way to determinate the DI of the blood cells in mimic in vitro microvascular environment.

Figure 6 shows a healthy RBC flowing along the axial position, $x$, of the microchannel with a hyperbolic-shaped contraction followed by a sudden expansion, which was subdivided into four sections to assess the DI evolution of the RBCs along the microchannel. Additionally, Fig. 6 shows the typical DI values found for healthy RBCs when exposed to a hyperbolic contraction (section $2, \sim 0.50$ ) followed by sudden expansion region (section $4, \sim 0.15$ ), under a controlled homogeneous extensional flow field (Lee et al. 2009; Yaginuma et al. 2013).

Using the set of data listed in Table 1, the DIs of the RBCs (control versus MNPs) were measured flowing along the centreline of the microchannel subdivided into four sections (cf. Figure 6), and the results are displayed in Fig. 7. Furthermore, to compare the

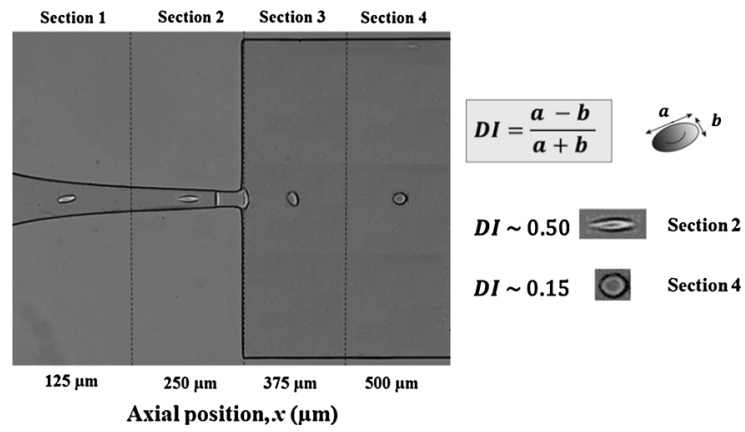

Fig. 6 Representation of a healthy RBC flowing along the axial position of a microchannel with a hyperbolic-shaped contraction followed by a sudden expansion, subdivided into four sections with equal distances between them $(125 \mu \mathrm{m})$. This approach allows the assessment of the RBCs DIs, as an indicator of the physiological effect of MNPs on the RBCs membranes. Furthermore, the DI equation is represented, as well as the typical values found for a healthy RBC subjected to a hyperbolic-shaped contraction (section 2) and to a recuperation section (section 4), under a controlled homogeneous extensional flow field deformability behaviour of the tested samples, the average shear rate and particle Reynolds number $\left(R e_{\mathrm{p}}\right)$, were calculated at four different sections as shown in Fig. 6 and Table 5. The average or pseudo shear rate is given by

$\bar{\gamma}=\frac{U}{D_{\mathrm{h}}}$

where $U$ is the mean velocity of the blood cells obtained at each region and $D_{\mathrm{h}}$ is the hydraulic diameter of each microchannel section (Müller et al. 2014). Additionally, the particle Reynolds number $\left(R e_{\mathrm{p}}\right)$ was defined as:

$\mathrm{Re}_{\mathrm{p}}=\frac{\rho \mathrm{UD} \mathrm{RBC}_{\mathrm{C}}}{\mu}$

where $D_{\mathrm{RBC}}$ is the diameter of the human $\mathrm{RBC}$ at rest, $\rho$ is the density of the Dextran 40 solution and $\mu$ is the shear viscosity of the same solution (Yaginuma et al. 2013). Table 5 lists the mean \pm standard deviation values calculated for the $R e_{\mathrm{p}}$ and average shear rate along the microchannel.

The $R e_{\mathrm{p}}$ is a useful tool to assess the behaviour of the flowing cells suspended in the working fluid, taking into account the inertial and viscous forces that occur through the interactions of the blood cells with the fluid. The results listed in Table 5 show that this interaction is higher in the section 2 , where the average shear rate is also higher, and decreases in the recuperation sections (sections 3 and 4), where the average shear rate becomes lower. Note that in microfluidic channels with a low aspect ratio $(h / w$, where $h$ refers to the depth $(20 \mu \mathrm{m})$ and $w$ refers to the width $(20-400 \mu \mathrm{m})$ of the microchannel), as those presented in our study, the RBC deformability is strongly affected by both extensional and shear flow conditions. Moreover, since the shear stress is usually reported in literature for the in vivo physiological flow conditions, the average shear rate was assessed and compared with values obtained in human vascular systems (Sadler 1998; Vennemann et al. 2007). In addition, the low standard deviation obtained between all the samples along the microchannel sections for the measured $R e_{\mathrm{p}}$ shows the maintenance of the same flow conditions in the two independent microfluidic experiments, which is crucial for the further comparison between the DI results. 

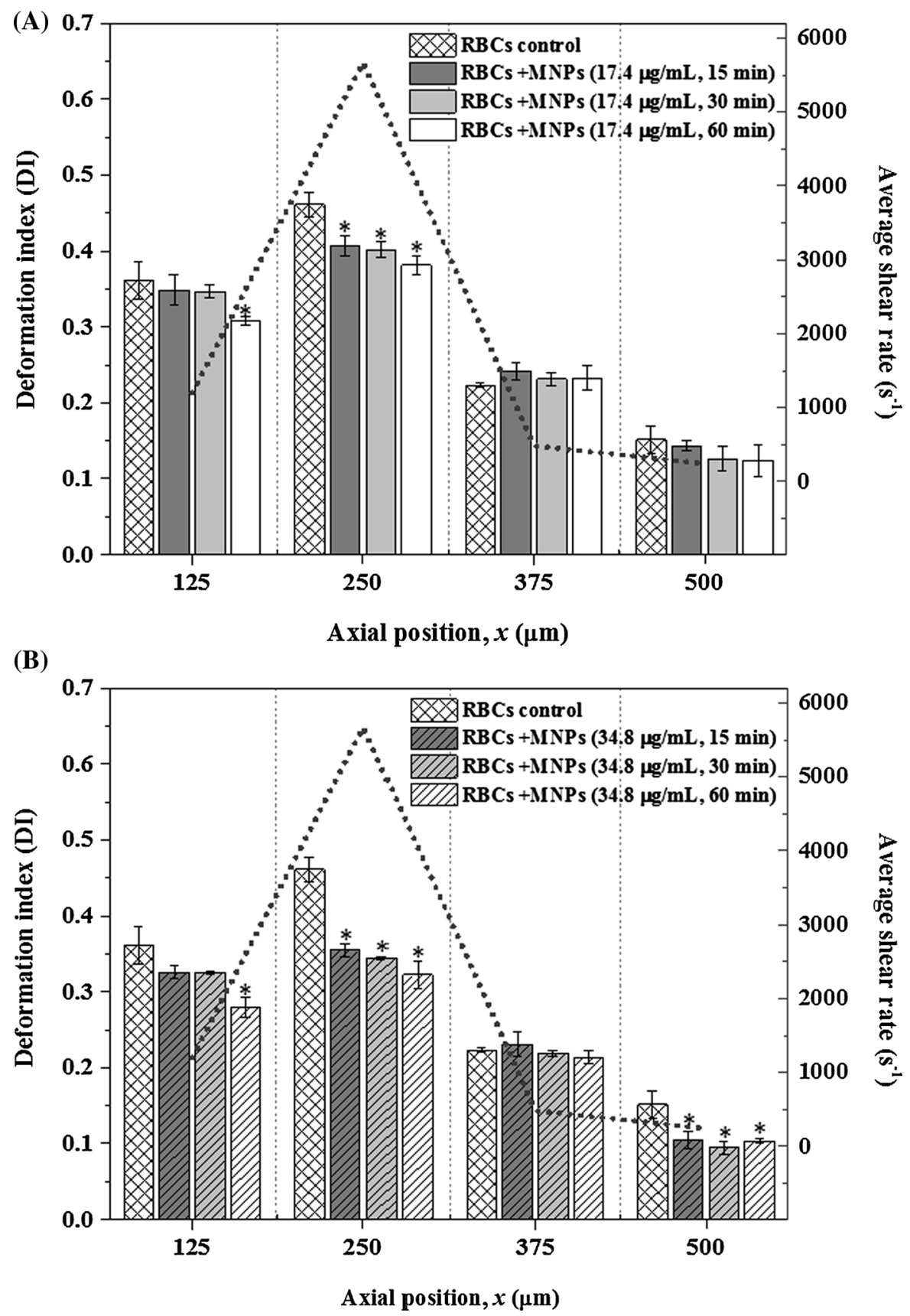

Fig. 7 Deformation index of blood cell flowing along the axial position of the hyperbolic channel, as well as the average shear rate at each hyperbolic region. a Control RBCs versus RBCs in contact with MNPs at a final concentration of $17.4 \mu \mathrm{g}\left(\mathrm{Fe}_{3} \mathrm{O}_{4}\right)$ / $\mathrm{mL}$ during 15, 30, and $60 \mathrm{~min}$; b Control RBCs versus RBCs in contact with MNPs at a final concentration of $34.8 \mu \mathrm{g}\left(\mathrm{Fe}_{3} \mathrm{O}_{4}\right)$ /

As expected, the maximum DI obtained with all tested RBCs samples was measured in the hyperbolic contraction region, where the width is minimum
$\mathrm{mL}$ during 15, 30, and $60 \mathrm{~min}$. Error bars show the standard deviation of two independent experiments $(n=112$ blood cells), and the asterisks $(*)$ represent statistical significance in comparison with control test for $p<0.05$ determined by Student's $t$ test

(section 2, axial position $250 \mu \mathrm{m}$ ) and the average shear rate is higher $\left(\sim 6000 \mathrm{~s}^{-1}\right)$. Indeed, this value of shear rate is very close to that of the arterioles in 
Table 5 Flow characteristics obtained for the four sections of the microchannel in the haemodynamic study

\begin{tabular}{lcccc}
\hline Flow characteristics & Section 1 & Section 2 & Section 3 & \multicolumn{1}{c}{ Section 4 } \\
\hline Particle Reynolds number & $0.08 \pm 0.02$ & $0.22 \pm 0.02$ & $0.04 \pm 0.00$ & $0.02 \pm 0.01$ \\
Average shear rate $\left(\mathrm{s}^{-1}\right)$ & $1198.66 \pm 83.82$ & $5670.50 \pm 731.29$ & $483.35 \pm 90.66$ & $241.16 \pm 65.97$ \\
\hline
\end{tabular}

Data represent mean \pm standard deviation for two independent experiments $(n=112$ blood cells)

the human vascular system $\left(\sim 8000 \mathrm{~s}^{-1}\right)$ (Sadler 1998; Vennemann et al. 2007) and thus, ideal to test the DI of RBCs at mimicked microvascular conditions and to detect changes in the RBC DIs, as an indicator of possible haematological disorders and nanoparticle-cell interactions. Overall, the higher DI, in section 2, was measured for the RBCs control (not exposed to MNPs), reaching a maximum DI of $0.46 \pm 0.02$. It is worth mentioning that this result is in good agreement with the results obtained by Lee et al. (2009) for healthy RBCs submitted to higher shear rates (0.42-0.60, for 0.6-9.0 Pa of extensional stress). Nevertheless, the DI results for the RBC samples in contact with MNPs show that the incubation time and MNPs amount present in the blood samples influence directly the maximum deformability of those blood cells, as shown by the statistical analysis in section 2 (maximum constriction region) of Fig. $7 \mathrm{a}$ and $\mathrm{b}$, where a significant decrease of deformability in comparison with the control test is observed. Hence, the DI results allow to conclude that the increase of the incubation time and the amount of MNPs lead to the decrease in DI or, in other words, to the increase of rigidity of those cells. This observation is even more obvious for the sample with the higher content of MNPs and exposure time $\left(34.8 \mu \mathrm{g}\left(\mathrm{Fe}_{3} \mathrm{O}_{4}\right) / \mathrm{mL}, 60 \mathrm{~min}\right)$, where the maximum DI detected (section 2) decreased to $0.32 \pm 0.02$, which is 1.44 times lower than the control DI $(0.46 \pm 0.02)$. The rigidity promoted by the MNPs into the RBC membranes is also observed in the recuperation section (section 4 , axial position $500 \mu \mathrm{m})$, where the average shear rate is minimum $\left(\sim 241 \mathrm{~s}^{-1}\right.$ ). In section 4 , and comparing the results obtained between the control sample and the RBCs samples that were placed in contact with the MNPs, it can be observed that RBCs incubated in the presence of MNPs become more rigid than the control RBCs, even when they are almost at rest. In the latter case, the control RBCs had ellipsoid shapes due to the applied shear flow, whereas RBCs in contact with MNPs had geometries closer to a circular shape which corresponds to the behaviour of rigid cells.

Recently, Lin et al. (2012) were able to measure the RBCs DI after the blood cells have been in contact with nanodiamond nanoparticles at several shear stress, using a laser aggregometer-deformometer device. This study has shown that large nanoparticles (100 nm average size) in contact with RBCs promote the reduction of the RBCs deformability, or the increase of their rigidity. Furthermore, using a confocal microscope and observing the blood samples in contact with the nanoparticles, the authors concluded that large nanodiamonds were located around the external environment of the RBCs, whereas smaller nanodiamonds ( $5 \mathrm{~nm}$ average size) penetrated inside the RBCs. As a result, it was established in this study that the deformability of RBCs decreases significantly for higher concentration of large nanodiamonds. This effect was ascribed to changes in the membranes of RBCs, probably caused by modifications in the structure and activity of their proteins, which may also lead to variations in the oxygenation-deoxygenation dynamics. More recently, similar conclusions were obtained by Curtis et al. (2015) through numerical simulations with hydrophilic nanoparticles with diameters ranging between 1 and $25 \mathrm{~nm}$, in a bilayer membrane (such as the RBCs membranes). The results have shown that hydrophilic nanoparticles with diameters greater than $2 \mathrm{~nm}$ become wrapped (the lipids head groups of a membrane form a vesicle around the nanoparticle), while smaller nanoparticles became embedded in the bilayer surface, penetrating into the membrane with minimal disruption to the structure. Note that none of these studies can be directly correlated with the deformability results obtained in this work, and thus proving that the DI changes observed are due to the MNPs wrap and uptake by the blood cells membranes. This direct correlation cannot be done since the size, shape and composition also play an important role in this cellular 
uptake. Therefore, further studies are required to clarify the complex interaction MNPs-RBCs. However, these works highlight the accuracy of the proposed microfluidic method, as a new and accurate tool to investigate the haemocompatibility of MNPs, where other more traditional analysis (such as haemolysis tests) cannot show any specific alteration.

\section{Conclusions}

MNPs for theranostic applications were synthesized, characterized and tested for magnetic hyperthermia and MRI. The different heating behaviour of the synthesized MNPs shows the importance of the temperature as a synthesis parameter that allows the growth of the magnetic core and subsequently allows the increment of the saturation magnetization, while the nanoparticle size remains in the single-domain region with superparamagnetic behaviour. As a result, the iron oxide nanoparticles were produced through a cost efficient and industry friendly scalable procedure, proven to be suitable for theranostics application. Nevertheless, in order to be suitable for biomedical applications, the synthesized MNPs should be haematological safe. Thus, and since there is a lack of studies in literature regarding this issue, a high-sensitivity microfluidic tool was tested to gain insights into the complex nanoparticle-RBCs membrane interaction. This novel toxicological and haematological concept was based on the proven fact that the natural deformability of the RBCs can be used as biophysical marker to assess haematological pathologies or disorders. Hence, for the first time, it is presented a microfluidic tool capable of evaluating with high accuracy the impact of multifunctional nanoparticles designed for theranostic applications in contact with RBCs, using the proved extensional flow approach to measure with high accuracy the RBC's DIs.

The traditional haematological test that was performed in this work by the conventional haemolysis analysis of the synthesised magnetic nanoparticles with diameter around $18 \mathrm{~nm}$ suggests that these nanoparticles are suitable for biomedical applications in environment conditions, without showing almost no haemolytic effect in the blood samples, even at the higher content of nanoparticles tested $(34.8 \mu \mathrm{g}$ $\left.\left(\mathrm{Fe}_{3} \mathrm{O}_{4}\right) / \mathrm{mL}\right)$. On the other hand, the applied microfluidic tool has shown that small MNPs can influence the rigidity of RBCs in a time-dependent manner, where other and more traditional biocompatibility tests (such as the haemolysis analysis) did not shown any influence. Moreover, it was demonstrated that this influence is directly related to the concentration of MNPs within the working fluid. In fact, the tested microfluidic tool has shown a higher sensitivity to detect small changes in the RBC deformability, which is unreachable using conventional techniques. Due to this unique ability, this is the first experimental test reported in the literature that subscribes the numerical work performed by Curtis et al. (2015), which points to the uptake of the surrounding MNPs by the RBC membranes, as the main reason for the increasing rigidity observed in the RBCs. In this recent numerical study, it was also predicted a dependency on the MNPs size, shape and composition with the RBC membrane interaction.

It should be noticed that to gain a further understanding of the haemorheological and biological interaction between MNPs and RBCs, other studies should be performed to complement this one. Nevertheless, the presented haematological and toxicological results confirm that it is highly desirable that blood properties, including the effect of the MNPs on the deformability of RBCs, and other rheological properties, should be controlled and tested together with the traditional biocompatible tests. These new achievements and the effective understanding of the interaction between MNPs and the blood cells will help to promote the use of nanomedicine to the next level. For instance, the MNPs-RBCs interactions revealed in this study can be in the future investigated as a new approach to deliver MNPs directly into the tumours, using the RBCs as biocarriers that naturally escape from the mononuclear phagocyte system. In fact, in a very recent review performed by Villa et al. (2016), they have shown the potentiality to use RBCs as supercarriers for drugs, biologicals and nanoparticles as advanced delivery systems. Hence, the proposed microfluidic methodology represents a new concept for the multidisciplinary nanomedicine field with proven benefits to evaluate the haemocompatibility of MNPs that are being developed for clinical applications.

Acknowledgments This work was financially supported by: Project POCI-01-0145-FEDER-006984 - Associate Laboratory 
LSRE-LCM funded by FEDER funds through COMPETE2020 Programa Operacional Competitividade e Internacionalização (POCI) - and by national funds through FCT - Fundação para a Ciência e a Tecnologia. R.O.R. acknowledges the Ph.D. scholarship SFRH/BD/97658/2013 Granted by FCT. A.M.T.S acknowledges the FCT Investigator 2013 Programme (IF/01501/ 2013), with financing from the European Social Fund and the Human Potential Operational Programme. M.B. would like to thank ERDF (European Regional Development Fund) under grant PO Norte CCDR-N/ON.2 Programme. J.G. also thanks the European Union's Seventh Framework Programme for research, technological development and demonstration under grant agreement no. 600375 .

\section{References}

Abkarian M, Faivre M, Horton R, Smistrup K, Best-Popescu CA, Stone HA (2008) Cellular-scale hydrodynamics. Biomed Mater 3:034011

Aphesteguy JC, Kurlyandskaya GV, de Celis JP, Safronov AP, Schegoleva NN (2015) Magnetite nanoparticles prepared by co-precipitation method in different conditions. Mater Chem Phys 161:243-249

Bañobre-López M, Teijeiro A, Rivas J (2013) Magnetic nanoparticle-based hyperthermia for cancer treatment. Rep Prac Oncol Radiother 18:397-400

Barick KC, Singh S, Bahadur D, Lawande MA, Patkar DP, Hassan PA (2014) Carboxyl decorated $\mathrm{Fe}_{3} \mathrm{O}_{4}$ nanoparticles for MRI diagnosis and localized hyperthermia. J Colloid Interface Sci 418:120-125

Baumgartner J, Bertinetti L, Widdrat M, Hirt AM, Faivre D (2013) Formation of magnetite nanoparticles at low temperature: from superparamagnetic to stable single domain particles. PLoS One 8:e57070

Curtis EM, Bahrami AH, Weikl TR, Hall CK (2015) Modeling nanoparticle wrapping or translocation in bilayer membranes. Nanoscale 7:14505-14514

De Haas-Kock DF, Buijen J, Pijls-Johannesma M, Lutgers L, Lammering G, van Mastrigt GA, De Ruysscher DK, Lambin P, van der Zee J (2009) Concomitant hyperthermia and radiation therapy for treating locally advanced rectal cancer. Cochrane Database Syst Rev 3:CD006269

Deatsch AE, Evans BA (2014) Heating efficiency in magnetic nanoparticle hyperthermia. J Magn Magn Mater 354:163-172

Faustino V, Pinho D, Yaginuma T, Calhelha R, Ferreira IFR, Lima R (2014) Extensional flow-based microfluidic device: deformability assessment of red blood cells in contact with tumor cells. BioChip J 8:42-47

Gallo J, Long NJ, Aboagye EO (2013) Magnetic nanoparticles as contrast agents in the diagnosis and treatment of cancer. Chem Soc Rev 42:7816-7833

Gallo J, Alam IS, Lavdas I, Wylezinska-Arridge M, Aboagye EO, Long NJ (2014) RGD-targeted MnO nanoparticles as T1 contrast agents for cancer imaging - the effect of PEG length in vivo. J Mater Chem B 2:868-876

Gossett DR et al (2012) Hydrodynamic stretching of single cells for large population mechanical phenotyping. PNAS 109:7630-7635
Grudzinski IP, Bystrzejewski M, Cywinska MA, Kosmider A, Poplawska M, Cieszanowski A, Ostrowska A (2013) Cytotoxicity evaluation of carbon-encapsulated iron nanoparticles in melanoma cells and dermal fibroblasts. J Nanopart Res 15:1835

Hao R, Xing R, Xu Z, Hou Y, Gao S, Sun S (2010) Synthesis, functionalization, and biomedical applications of multifunctional magnetic nanoparticles. Adv Mater 22:27292742

Hayashi K et al (2013) Superparamagnetic nanoparticle clusters for cancer theranostics combining magnetic resonance imaging and hyperthermia treatment. Theranostics 3:366-376

Hervault A, Thanh NTK (2014) Magnetic nanoparticle-based therapeutic agents for thermo-chemotherapy treatment of cancer. Nanoscale 6:11553-11573

Hocaoglu I et al (2015) Cyto/hemocompatible magnetic hybrid nanoparticles $\left(\mathrm{Ag} 2 \mathrm{~S}-\mathrm{Fe}_{3} \mathrm{O}_{4}\right)$ with luminescence in the near-infrared region as promising theranostic materials. Colloids Surf B 133:198-207

Jordan A, Scholz R, Wust P, Fähling H, Roland F (1999) Magnetic fluid hyperthermia (MFH): cancer treatment with AC magnetic field induced excitation of biocompatible superparamagnetic nanoparticles. J Magn Magn Mater 201:413-419

Kang YJ, Ha Y-R, Lee S-J (2016) Deformability measurement of red blood cells using a microfluidic channel array and an air cavity in a driving syringe with high throughput and precise detection of subpopulations. Analyst. doi:10.1039/ C5AN01988E

Khorsand ZA, Majid WH, Abrishami ME, Yousefi R (2011) $\mathrm{X}$-ray analysis of $\mathrm{ZnO}$ nanoparticles by Williamson-Hall and size-strain plot methods. Solid State Sci 13:251-256

Kolen'ko YV et al (2014) Large-scale synthesis of colloidal $\mathrm{Fe}_{3} \mathrm{O}_{4}$ nanoparticles exhibiting high heating efficiency in magnetic hyperthermia. J Phys Chem C 118:8691-8701

Lee SS, Yim Y, Ahn KH, Lee SJ (2009) Extensional flow-based assessment of red blood cell deformability using hyperbolic converging microchannel. Biomed Microdevices 11:1021-1027

Lima $R$ et al (2008) In vitro blood flow in a rectangular PDMS microchannel: experimental observations using a confocal micro-PIV system. Biomed Microdevices 10:153-167

Lin YC et al (2012) The influence of nanodiamond on the oxygenation states and micro rheological properties of human red blood cells in vitro. J Biomed Opt 17:101512

Lu A-H, Salabas EL, Schüth F (2007) Magnetic nanoparticles: synthesis, protection, functionalization, and application. Angew Chem Int Ed 46:1222-1244

Mahmoudi M, Sant S, Wang B, Laurent S, Sen T (2011) Superparamagnetic iron oxide nanoparticles (SPIONs): development, surface modification and applications in chemotherapy. Adv Drug Deliv Rev 63:24-46

Mayer A, Vadon M, Rinner B, Novak A, Wintersteiger R, Frohlich E (2009) The role of nanoparticle size in hemocompatibility. Toxicology 258:139-147

Müller K, Fedosov DA, Gompper G (2014) Margination of micro- and nano-particles in blood flow and its effect on drug delivery. Scientific Reports 4:4871

Obaidat I, Issa B, Haik Y (2015) Magnetic properties of magnetic nanoparticles for efficient hyperthermia. Nanomaterials 5:63 
Oliveira MSN, Alves MA, Pinho FT, McKinley GH (2007) Viscous flow through microfabricated hyperbolic contractions. Exp Fluids 43:437-451

Pinho D, Yaginuma T, Lima R (2013) A microfluidic device for partial cell separation and deformability assessment. BioChip J 7:367-374

Presa P, Luengo Y, Multigner M, Costo R, Morales MP, Rivero G, Hernando A (2012) Study of heating efficiency as a function of concentration, size, and applied field in $\gamma$ Fe2O3 nanoparticles. J Phys Chem C 116:25602-25610

Rivas J, Bañobre-López M, Piñeiro-Redondo Y, Rivas B, López-Quintela MA (2012) Magnetic nanoparticles for application in cancer therapy. J Magn Magn Mater 324:3499-3502

Rodrigues RO, Costa H, Lima R, Amaral JS (2015) Simple methodology for the quantitative analysis of fatty acids in human red blood cells. Chromatographia 78:1271-1281

Ruiz A, Morais PC, Azevedo RB, Lacava ZGM, Villanueva A, Morales MP (2014) Magnetic nanoparticles coated with dimercaptosuccinic acid: development, characterization, and application in biomedicine. J Nanopart Res 16:2589

Sabo A, Jakovljevic V, Stanulovic M, Lepsanovic L, Pejin D (1993) Red blood cell deformability in diabetes mellitus: effect of phytomenadione. Int J Clin Pharmacol Ther Toxicol 31:1-5

Sadler JE (1998) Biochemistry and genetics of von Willebrand factor. Annu Rev Biochem 67:395-424

Saraswathy A et al (2014) Citrate coated iron oxide nanoparticles with enhanced relaxivity for in vivo magnetic resonance imaging of liver fibrosis. Colloids Surf B 117:216-224

Sharifi I, Shokrollahi H, Amiri S (2012) Ferrite-based magnetic nanofluids used in hyperthermia applications. J Magn Magn Mater 324:903-915

Suwanarusk R, Cooke BM, Dondorp AM, Silamut K, Sattabongkot J, White NJ, Udomsangpetch R (2004) The deformability of red blood cells parasitized by Plasmodium falciparum and P. vivax. J Infect Dis 189:190-194

Szekeres M et al (2015) Hemocompatibility and biomedical potential of Poly (Gallic Acid) coated iron oxide nanoparticles for theranostic use. J Nanomed Nanotechnol 6:1000252

Tan J, Thomas A, Liu Y (2012) Influence of red blood cells on nanoparticle targeted delivery in microcirculation. Soft Matter 8:1934-1946

Thomas A, Tan J, Liu Y (2014) Characterization of nanoparticle delivery in microcirculation using a microfluidic device. Microvasc Res 94:17-27

Vennemann P, Lindken R, Westerweel J (2007) In vivo wholefield blood velocity measurement techniques. Exp Fluids 42:495-511

Villa CH, Anselmo AC, Mitragotri S, Muzykantov V (2016) Red blood cells: supercarriers for drugs, biologicals, and nanoparticles and inspiration for advanced delivery systems. Adv Drug Deliv Rev. doi:10.1016/j.addr.2016.02. 007

Wang Q, Shen M, Zhao T, Xu Y, Lin J, Duan Y, Gu H (2015) Low toxicity and long circulation time of polyampholytecoated magnetic nanoparticles for blood pool contrast agents. Scientific Reports 5:7774

Weinstein JS et al (2010) Superparamagnetic iron oxide nanoparticles: diagnostic magnetic resonance imaging and potential therapeutic applications in neurooncology and central nervous system inflammatory pathologies, a review. J Cereb Blood Flow Metab 30:15-35

Yaginuma T, Oliveira MSN, Lima R, Ishikawa T, Yamaguchi T (2013) Human red blood cell behavior under homogeneous extensional flow in a hyperbolic-shaped microchannel. Biomicrofluidics 7:054110

Yaylali YT et al (2013) Increased red blood cell deformability and decreased aggregation as potential adaptive mechanisms in the slow coronary flow phenomenon. Coron Artery Dis 24:11-15

Zavisova V et al (2015) The cytotoxicity of iron oxide nanoparticles with different modifications evaluated in vitro. J Magn Magn Mater 380:85-89

Zheng Y, Shojaei-Baghini E, Azad A, Wang C, Sun Y (2012) High-throughput biophysical measurement of human red blood cells. Lab Chip 12:2560-2567 